\title{
Wavelength-Routed Optical Networks: Linear Formulation, Resource Budgeting Tradeoffs, and a Reconfiguration Study
}

\author{
Dhritiman Banerjee and Biswanath Mukherjee, Member, IEEE
}

\begin{abstract}
We present algorithms for the design of optimal virtual topologies embedded on wide-area wavelength-routed optical networks. The physical network architecture employs wavelength-conversion-enabled wavelength-routing switches (WRS) at the routing nodes, which allow the establishment of circuit-switched all-optical wavelength-division multiplexed (WDM) channels, called lightpaths. We assume packet-based traffic in the network, such that a packet travelling from its source to its destination may have to multihop through one or more such lightpaths.

We present an exact integer linear programming (ILP) formulation for the complete virtual topology design, including choice of the constituent lightpaths, routes for these lightpaths, and intensity of packet flows through these lightpaths. By minimizing the average packet hop distance in our objective function and by relaxing the wavelength-continuity constraints (i.e., assuming wavelength converters at all nodes), we demonstrate that the entire optical network design problem can be considerably simplified and made computationally tractable.

Although an ILP may take an exponential amount of time to obtain an exact optimal solution, we demonstrate that terminating the optimization within the first few iterations of the branch-and-bound method provides high-quality solutions. We ran experiments using the CPLEX optimization package on the NSFNET topology, a subset of the PACBELL network topology, as well as a third random topology to substantiate this conjecture.

Minimizing the average packet hop distance is equivalent to maximizing the total network throughput under balanced flows through the lightpaths. The problem formulation can be used to design a balanced network, such that the utilizations of both transceivers and wavelengths in the network are maximized, thus reducing the cost of the network equipment. We analyze the tradeoffs in budgeting of resources (transceivers and switch sizes) in the optical network, and demonstrate how an improperly designed network may have low utilization of any one of these resources. We also use the problem formulation to provide a reconfiguration methodology in order to adapt the virtual topology to changing traffic conditions.
\end{abstract}

Index Terms-Integer linear program, lightpath, optical network, optimization, reconfigurability, resource budgeting, virtual topology, wavelength routing, WDM.

Manuscript received January 2, 1997; revised February 3, 1998; approved by IEEE/ACM TRANSACTIONS ON NETWORKING Editor G. Sasaki. This work was supported by the National Science Foundation under Grant NCR-92-05755 and Grant NCR-95-08239.

D. Banerjee is with Bell Laboratories, Lucent Technology, Holmdel, NJ 07733 USA (e-mail: banerjed@lucent.com).

B. Mukherjee is with the Department of Computer Science, University of California, Davis, CA 95616 USA (e-mail: mukherje@pepper.cs.ucdavis.edu).

Publisher Item Identifier S 1063-6692(00)09126-3.

\section{INTRODUCTION}

$\mathbf{W}$ AVELENGTH-DIVISION multiplexing (WDM) provides the ability to carve up the huge optical bandwidth available in fiber into lower-capacity noninterfering wavelengths (channels), based on which multichannel lightwave networks can be built [4], [17]. In this approach, each node needs to operate only at the speed of an individual channel. Each optical channel, called a lightpath [6], may be used to carry information across multiple fiber links, without the data undergoing electronic packet processing at intermediate nodes in the network.

We assume that the reader is knowledgeable about the general architecture of a wide-area wavelength-routed optical network. References [6], [15], [4], [18], [17], [20] provide a good introduction to the physical architecture of the optical network and the constituent optimization problems in designing an optimal lightpath-based optical network for packet-switched traffic. Prototype networks based on this architecture are being constructed as part of the MONET, AON, NTONC, RACE-MWTN, and other projects (see [9]-[11]). In addition, several companies such as Tellium, Ciena, Nortel, Corvis, Lucent, and Sycamore Networks have announced products that are being used by companies such as Level 3 Communications, Qwest, UUNET, and Williams Communication to build the next generation of optical network infrastructures based on similar wavelength-routed architectures.

The physical network architecture employs a wavelength-routing switch (WRS) equipped with wavelength convertors at the routing nodes. Recent studies in network design suggest the use of wavelength conversion in the network in order to overcome compatibility issues across equipment from different vendors. This viewpoint has given rise to the increasing popularity of "opaque network" designs. This assumption also relaxes the wavelength-continuity constraint in the network. ${ }^{1}$

In this paper, we present the design of a lightpath-based optical network as an integer linear programming (ILP) optimization problem, and use the problem formulation to seek an optimal network design. The formulation presented here is a modified version of the problem formulation given in [15] which,

${ }^{1}$ In the absence of wavelength conversion in the network, a lightpath would need to be on the same wavelength across all the fibers that it traverses. This adds to the complexity of the optimization problem (as we shall demonstrate). 
though complete, contained nonlinear equations and was difficult to solve exactly. In this paper, we revise the optimization problem from a nonlinear set of equations to a linear set of equations together with a few integer constraints. We used the branch-and-bound methodology in the CPLEX optimization package [1] for solving the integer set of equations; however, we demonstrate that terminating the optimization within a few iterations of the branch-and-bound method continues to provide high-quality solutions. We also present a number of engineering techniques to make the network design problem more computationally tractable, thus allowing the network designer to balance between the time taken to obtain a solution and the optimality of the solution.

We simplify the objective function to minimize the average packet hop distance ${ }^{2}$ (which is inversely proportional to the network throughput under balanced network flows and which is a linear objective function). By relaxing the wavelength-continuity constraints (i.e., assuming wavelength converters at all nodes), we demonstrate that the entire optical network design problem can be considerably simplified. If the channel (lightpath) capacity is $C$, the number of channels is $L$, and the average packet hop distance is $H$, then the network throughput can be approximated by

$$
T \leq \frac{C L}{H}
$$

under balanced loading of all channels. Therefore, minimizing $H$ is equivalent to maximizing the network throughput. ${ }^{3}$ We develop an ILP formulation to minimize $H$, the average packet hop distance, for a virtual-topology-based wavelength-routed network. The ILP formulation provides a complete specification to the virtual topology design, routing of the constituent lightpaths, and intensity of packet flows though the lightpaths.

Designing an optical network for packet data has been studied before [5], [6], [18], [22]. While many research papers have largely ignored the implications of using packet-traffic data in their problem formulation, we believe that using packet data for the traffic matrix in the problem formulation significantly increases the usefulness of the solution.

Other researchers have also formulated an integer linear program to solve the virtual topology design problem [18]. However, the complexity of their formulation seemed to limit its applicability for only small networks. By simplifying the objective function, our approach is able to solve larger network design problems in reasonable time. Our experiments also suggest that terminating the ILP optimization after a few iterations of the branch-and-bound method continues to provide high-quality solutions, which makes our approach more practical for use in a real network.

Section II formulates the problem mathematically as an ILP. This section also discusses some of our underlying assumptions

\footnotetext{
${ }^{2}$ The average packet hop distance is defined as the number of lightpaths that a packet has to traverse on average, and is a function of the virtual topology.

${ }^{3}$ In a non-WDM network, the number of channels is given by $M$, the number of fiber links in the network. Therefore, the non-WDM network throughput is given by $T_{p} \leq\left(C M / H_{p}\right)$, where $H_{p}$ denotes the average packet hop distance in the non-WDM network.
}

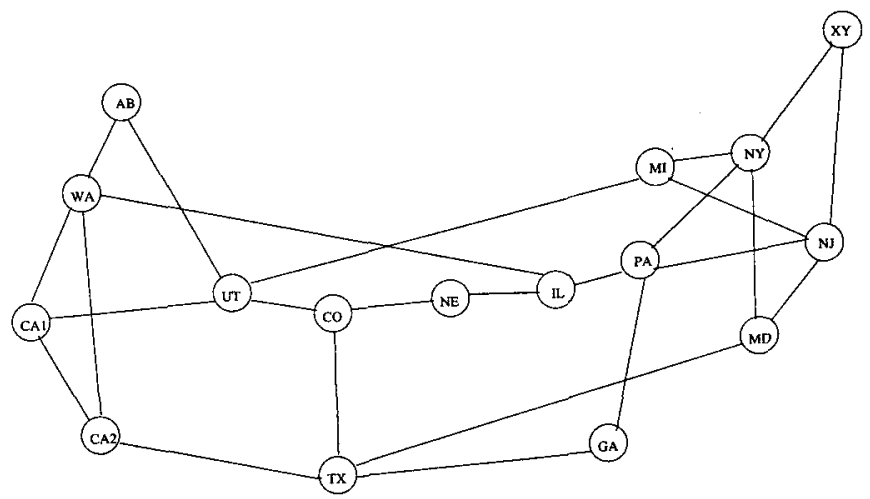

Fig. 1. NSFNET T1 backbone.

that can drastically reduce the size of the problem, and consequently minimize the running time of the solution. Section III presents two simple heuristics with fast running times whose performance compares favorably with the performance of the optimized solution (obtained from the ILP solution). Heuristics become important when the size of the problem becomes larger than an ILP solver can handle, or when the optimization needs to be achieved in real time; we demonstrate that the proposed heuristics perform well with respect to the optimal bound (viz. ILP output). Section IV examines tradeoffs that affect the quality of the solution, and discusses resource-budgeting strategies for transmission/reception as well as switching equipment. This approach allows the design of a balanced network, which can provide "optimal" network throughput along with high utilization of both transceivers and wavelengths. Section V proposes an algorithm which can be used for virtual topology reconfiguration. The proposed algorithm computes a new virtual topology from an existing virtual topology, such that the new virtual topology is optimal with respect to the changing traffic patterns; among all such optimal virtual topologies, the algorithm selects the topology which is "closest" in structure to the current virtual topology, thus minimizing the number of switch retunings during the transition period, as well as minimizing both the number of disrupted lightpaths and the reconfiguration time. Section VI provides numerical simulation results obtained from solving the above formulation for the NSFNET topology (see Fig. 1), the PACBELL topology (see Fig. 2), and a random topology with 20 nodes. Section VII concludes the paper.

\section{Problem SPECIFICATION}

Much of the notation and the constraint equations used in this section are borrowed from [15]. They are repeated here for completeness of the problem specification. New material, relative to that in [15], include a new linear objective function (2), a constraint to bound the lightpath length (17), a constraint to bound the maximum loading per channel (26), a constraint to incorporate the physical topology as part of the virtual topology (16), all of the simplifying assumptions in Section II-B (to make the problem computationally tractable), and several other generalizations (multiple fibers between nodes, multiple lightpaths between node pairs, no bidirectional lightpaths, etc.). 


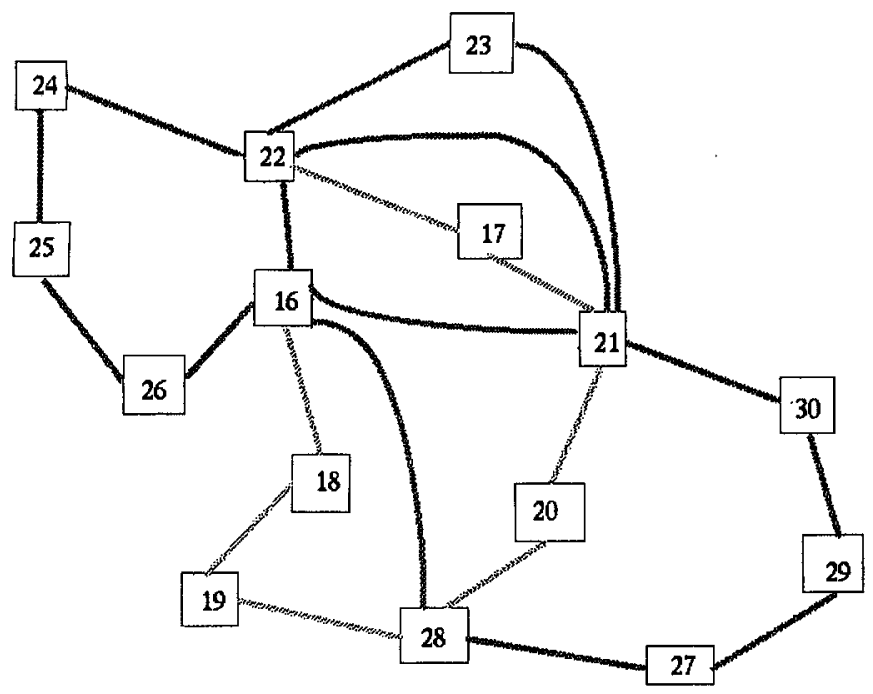

Fig. 2. A portion of the PACBELL backbone.

\section{A. Linear Formulation}

We formulate the problem as an optimization problem, using principles from multicommodity flow for physical routing of lightpaths and traffic flow on the virtual topology, and using the following notation:

- $s$ and $d$ used as subscript or superscript denote source and destination of a packet, respectively.

- $i$ and $j$ denote originating and terminating nodes, respectively, in a lightpath.

- $m$ and $n$ denote endpoints of a physical link that might occur in a lightpath.

Given:

- Number of nodes in the network $=N$.

- Maximum number of wavelengths per fiber $=W$ (a system-wide parameter).

- Physical topology $P_{m n}$ denotes the number of fibers interconnecting node $m$ and node $n . P_{m n}=0$ for nodes which are not physically adjacent to each other. $P_{m n}=P_{n m}$ indicates that there are equal number of fibers joining two nodes in different directions. Note that there may be more than one fiber link connecting adjacent nodes in the network. $\sum_{m, n} P_{m n}=M$ denotes the total number of fiber links in the network.

- Fiber length matrix, viz. fiber distance $d_{m n}$ from node $m$ to node $n$. For simplicity in expressing packet delays, $d_{m n}$ is expressed as a propagation delay (in time units). Note that $d_{m n}=d_{n m}$, and $d_{m n}=\infty$ if $P_{m n}=0$.

- Shortest path delay matrix $D$ where $D_{s d}$ denotes the delay (sum of propagation delays only) over the shortest path between nodes $s$ and $d$.

- Lightpath length bound $\alpha, 1 \leq \alpha<\infty$, bounds the delay over a lightpath (and hence the length of the lightpath) between two nodes $i$ and $j$, with respect to the shortest path delay $D_{i j}$ between them, i.e., the maximum permissible propagation delay over the lightpath between the two nodes $i$ and $j$ is $\alpha \times D_{i j}$.

- Number of transmitters at node $i=T_{i}\left(T_{i} \geq 1\right)$. Number of receivers at node $i=R_{i}\left(R_{i} \geq 1\right)$. In general, we would assume that $T_{i}=R_{i}, \forall i$, although this is not a strict requirement.

- Traffic matrix $\Lambda_{s d}$ denotes the average rate of traffic flow (in packets/s) from node $s$ to node $d$, with $\Lambda_{s s}=0$ for $s$, $d=1,2, \cdots, N$.

- Capacity of each channel $=C$ (normally expressed in bits/s, but converted to units of packets/s by knowing the mean packet length).

- Maximum loading per channel $=\beta, 0<\beta<1$. $\beta$ restricts the queueing delay on a lightpath from getting unbounded by avoiding excessive link congestion. We do not incorporate queueing delays explicitly in the problem for mulation, under the assumption that they are negligible for suitably chosen values of $\beta$. Also, previous results [15] indicate that queueing delays are negligibly small compared to propagation delays for a large network setting as in Fig. 1, except under extremely heavy loading. While queuing delays may be large in today's (congested) backbone networks, the use of high-capacity WDM links along with silicon routers in the switching nodes should considerably alleviate this problem in future networks.

\section{Variables:}

- Virtual topology: The variable $V_{i j}$ denotes the number of lightpaths from node $i$ to node $j$ in the virtual topology. Note that the current formulation is general, since lightpaths are not necessarily assumed to be bidirectional, i.e., $V_{i j}=0 \nRightarrow V_{j i}=0$. Moreover, there may be multiple lightpaths between the same source-destination pair, i.e., $V_{i j}>1$, for the case when traffic between nodes $i$ and $j$ is greater than a single lightpath's capacity $(C)$.

- Traffic routing: The variable $\lambda_{i j}^{s d}$ denotes the traffic flowing from node $s$ to node $d$ and employing $V_{i j}$ as an intermediate virtual link. Note that traffic from node $s$ to node $d$ may be "bifurcated," with different components flowing through different sets of lightpaths.

- Physical topology route: The variable $p_{m n}^{i j}$ denotes the number of lightpaths between nodes $i$ and $j$ being routed though fiber link $m n$.

Objective: Optimality Criterion:

$$
\text { Mininize: } \frac{1}{\sum_{s, d} \Lambda_{s d}} \sum_{i, j} \sum_{s, d} \lambda_{i j}^{s d}
$$

The objective function minimizes the average packet hop distance in the network. This is a linear objective function because $\left(\sum_{i, j} \sum_{s, d} \lambda_{i j}^{s d}\right)$ is a linear sum of variables, while $\sum_{s, d} \Lambda_{s d}$ is a constant for a given traffic matrix. The two objective functions used in [15] were: 1) minimization of the average packet delay over the network; and 2) minimization of the maximum traffic flow in a lightpath; both were nonlinear.

\section{Constraints:}

- On virtual topology connection matrix $V_{i j}$ :

$$
\begin{gathered}
\sum_{j} V_{i j} \leq T_{i} \quad \forall i \\
\sum_{i} V_{i j} \leq R_{j} \quad \forall j \\
i n t \quad V_{i j} .
\end{gathered}
$$


The above equations ensure that the number of lightpaths emerging from a node is constrained by the number of transmitters at that node, while the number of lightpaths terminating at a node is constrained by the number of receivers at that node. $V_{i j}$ variables can only hold integer values. If $V_{i j}$ has a value greater than 1 , it means that there is more than one lightpath between the particular source-destination pair. These lightpaths may follow the same route or different routes through the network.

- On physical route variables $p_{m n}^{i j}$ :

$$
\begin{aligned}
& \sum_{m} p_{m k}^{i j}=\sum_{n} p_{k n}^{i j} \quad \text { if } k \neq i, j \\
& \sum_{n} p_{i n}^{i j}=V_{i j} \\
& \sum_{m} p_{m j}^{i j}=V_{i j} \\
& \sum_{i, j} p_{m n}^{i j} \leq W \times P_{m n} \\
& i n t \quad p_{m n}^{i j} .
\end{aligned}
$$

Equations (6)-(8) are multicommodity-flow-based equations governing the routing of lightpaths from source to destination. Equation (9) ensures that the number of lightpaths flowing through a fiber link does not exceed $W$. Note, however, that the equations do not ensure the wavelength-continuity constraint (under which the lightpath is assigned the same wavelength on all the fiber links through which it passes). Since our nodal architecture provides wavelength conversion, the wavelength-continuity constraint is unnecessary, although it has been generally observed that sparse wavelength conversion is sufficient in most networks [7], [8], [21]. Integrality constraints ensure that the $p_{i j}^{m n}$ variables hold only integer values.

- On virtual topology traffic variables $\lambda_{i j}^{s d}$ :

$$
\begin{aligned}
\sum_{j} \lambda_{s j}^{s d} & =\Lambda_{s d} \\
\sum_{i} \lambda_{i d}^{s d} & =\Lambda_{s d} \\
\sum_{i} \lambda_{i k}^{s d} & =\sum_{j} \lambda_{k j}^{s d}, \quad \text { if } k \neq s, d \\
\lambda_{i j}^{s d} & \leq \Lambda_{s d} \times V_{i j} \\
\sum_{s, d} \lambda_{i j}^{s d} & \leq \beta V_{i j} C .
\end{aligned}
$$

Equations (11)-(13) are multicommodity-flow equations governing the flow of traffic through the virtual topology. Note that the routing of traffic from a given source to a given destination may be bifurcated. Equation (14) ensures that traffic can only flow through an existing lightpath, while (15) specifies the capacity constraint in the formulation.

- Optional constraints:

1) Physical topology as a subset of the virtual topology:

$$
P_{m n}=1 \Rightarrow V_{m n} \geq 1, p_{m n}^{m n} \geq 1 \text {. }
$$

2) Bound lightpath length:

$$
\begin{gathered}
\sum_{m, n} p_{m n}^{i j} * d_{m n} \leq \alpha \times D_{i j} \times K \\
\text { for } K \text { alternate paths. }
\end{gathered}
$$

Equations (16) and (17) are optional constraints, and may be incorporated to ensure bounded packet delays in the network. These equations significantly reduce the solution space of the problem, and facilitate quicker convergence toward the optimal solution. This engineering approach enables us to obtain good solutions for large networks quickly.

Equation (16) embeds the physical topology as a subset of the virtual topology, i.e., every link in the physical topology is also a lightpath in the virtual topology, in addition to which there are lightpaths which span multiple fiber links. This approach for choosing lightpaths can satisfy packets with the tightest delay constraints [18]. The lightpaths corresponding to the physical topology may also be used to route network control messages efficiently, and this approach can simplify network management. For these equations to be valid, $T_{n} \geq \delta_{n}^{o}$ and $R_{n} \geq \delta_{n}^{i}$, where $\delta_{n}^{o}$ and $\delta_{n}^{i}$ denote the physical number of fibers emerging from and terminating at node $n$, respectively.

Equation (17) may be used to restrict the enumerated $p_{m n}^{i j}$ variables to be only among those present in $K$ alternate shortest paths from $i$ to $j$, where $K \geq 1$. This prevents long and convoluted lightpaths, i.e., lightpaths with an unnecessarily long route instead of a much shorter route from occurring. The value of $K$ may be selected by the network designer.

\section{B. Simplifying Assumptions}

This section outlines some simplifying assumptions to make the problem more tractable.

- Wavelength-continuity constraints for a lightpath are relaxed in the current formulation, which only ensures that the total number of lightpaths routed through a fiber is less than or equal to $W$. Adding wavelength-continuity constraints to the above set of equations significantly increases the complexity of the problem, ${ }^{4}$ e.g., if the variable $c_{k}^{i j}=1$ signifies that a lightpath from node $i$ to node $j$ is assigned the wavelength $k$ (where $k=1,2, \cdots, W$ ), the relevant equations are as follows:

$$
\begin{gathered}
\sum_{k} c_{k}^{i j}=V_{i j} \\
\sum_{i j} p_{m n}^{i j} . c_{k}^{i j} \leq 1 \quad \forall m, n, k .
\end{gathered}
$$

Equation (19) is nonlinear because it involves the product of two variables. As mentioned before, we intentionally ignore the wavelength assignment of lightpaths in the current problem formulation since we assume that wavelength convertors are available at the routing nodes [14]. Moreover, it has also been observed that sparse wavelength conversion in the switching nodes

${ }^{4}$ The wavelength-assignment problem has been demonstrated to be NP-complete [6]. 
is sufficient to achieve nearly the same performance as full conversion[7], [8], [21].

- Queueing delays are also intentionally ignored, partly to simplify (linearize) the objective function, and also because it has been observed that the propagation delay dominates the overall network delay in nationwide optical networks with highspeed transmission equipment and moderate loads. The advent of multi-gigabit silicon-based routers (as opposed to slow software-based routers) to be deployed shortly in the internet backbone also justifies this assumption. The exact optimization function for delay minimization is as follows [15]:

$$
\begin{aligned}
& \text { Minimize } \\
& \sum_{i j}\left(\sum_{s d} \lambda_{i j}^{s d}\left[\sum_{m n} p_{m n}^{i j} * d_{m n}+\frac{1}{C-\sum_{s d} \lambda_{i j}^{s d}}\right]\right) .
\end{aligned}
$$

This is a nonlinear equation because it involves the product of two variables, $\lambda_{i j}^{s d}$ and $p_{m n}^{i j}$, and also because the $1 /(C-$ $\sum_{s d} \lambda_{i j}^{s d}$ ) component leads to nonlinearities.

- The number of variables and equations in the formulation are reduced. The number of variables and equations in the original problem formulation grows as $O\left(N^{4}\right)$, and can very easily overwhelm today's state-of-the-art computing facilities. To make the problem more tractable, we reduce the number of constraints by pruning the search space. Pruning is based on tracking a limited number of alternate shortest paths, denoted by $K$, between source-destination pairs, such that the selected routes are within a constant factor $(\alpha \geq 1)$ of the shortest-path distance between the given source-destination pair. We assume that traffic flow will only use the lightpaths which interconnect nodes present in these alternate paths, i.e., all values of $\lambda_{i j}^{s d}$ are not enumerated. Likewise, lightpaths may only be routed through one of a few permissible routes, i.e., all possible values of $p_{m n}^{i j}$ are not enumerated. Since these assumptions are incorporated during the generation of the problem formulation, it helps reduce the total number of equations and variables. The amount of pruning (hence, the value of $K$ ) required is a function of the size of the problem that can be solved in "reasonable time" by the chosen optimization package.

To understand the pruning process, let us consider the NSFNET topology in Fig. 1. If we consider two alternate shortest paths between any source-destination pair, then the two alternate paths from Node (CA1) to Node (IL) may be CA1-UT-CO-NE-IL and CA1-WA-IL. Then, the enumerated variables for lightpath routing are as follows:

$p_{C A 1, U T}^{C A 1, I L}, p_{U T, C O}^{C A 1, I L}, p_{C O, N E}^{C A 1, I L}, p_{N E, I L}^{C A 1, I L}, p_{C A 1, W A}^{C A 1, I L}, p_{W A, N E}^{C A 1, I L}$

Likewise, the enumerated variables for packet routing are as follows:

$$
\begin{aligned}
& \lambda_{C A 1, U T}^{C A 1, I L}, \lambda_{C A 1, C O}^{C A 1, I L}, \lambda_{C A 1, N E}^{C A 1, I L}, \lambda_{C A 1, I L}^{C A 1, I L}, \lambda_{U T, C O}^{C A 1, I L}, \\
& \lambda_{U T, N E}^{C A 1, I L}, \lambda_{U T, I L}^{C A 1, I L}, \lambda_{C O, N E}^{C A 1, I L}, \lambda_{C O, I L}^{C A 1, I L}, \lambda_{N E, I L}^{C A 1, I L} \\
& \lambda_{C A 1, W A}^{C A 1, I L}, \lambda_{W A, I L}^{C A 1, I L} .
\end{aligned}
$$

- The current formulation allows bifurcated routing of packet traffic. To specify nonbifurcated routing of traffic, we use new variables $\gamma_{i j}^{s d}$ which are only allowed to take binary values, and the equations are suitably modified. Under nonbifurcated routing, (11)-(15) become

$$
\begin{aligned}
\gamma_{i j}^{s d} & \in 0,1 \\
\sum_{j} \gamma_{s j}^{s d} & =1 \\
\sum_{i} \gamma_{i d}^{s d} & =1 \\
\sum_{i} \gamma_{i k}^{s d} & =\sum_{j} \gamma_{k j}^{s d} \quad \text { if } k \neq s, d \\
\gamma_{i j}^{s d} & \leq V_{i j} \\
\sum_{s, d} \gamma_{i j}^{s d} \times \Lambda_{s d} & \leq \beta V_{i j} C .
\end{aligned}
$$

The objective function becomes

$$
\text { Minimize : } \frac{1}{\sum_{s, d} \Lambda_{s d}}\left(\sum_{i, j} \sum_{s, d}, \gamma_{i j}^{s d} \times \Lambda_{s d}\right) .
$$

We only used bifurcated routing in our experiments, since it was found that nonbifurcated routing of packet traffic significantly increased the running time of the optimization solution because of the additional integer constraints. The increase in running time is also due to the computation of the product terms in (26) and in the modified objective function.

\section{HEURISTIC APPROACHES}

This section presents two heuristic approaches that allow us to solve large problem instances of the virtual-topology design problem, in order to minimize the average packet hop distance. Heuristics become important when the problem formulation becomes large due to increase in the physical size of the network, and becomes difficult to solve by traditional LP methods due to computational constraints. Results of these heuristics compare favorably with the optimal result obtained by solving the exact problem formulation.

- Maximizing Single-Hop Traffic. This simple and greedy heuristic attempts to establish lightpaths between source-destination pairs with the highest $\Lambda_{s d}$ values, subject to constraints on the number of transceivers at the two end nodes, and the availability of a wavelength in some path connecting the two end nodes. It is the same as the heuristic logical topology design (HLDA) algorithm in [18], and was also proposed in earlier research [12].

- Maximizing Multihop Traffic. In a packet-switched network, the traffic carried by a link may include forwarded traffic as well as traffic originating from the attached node. Intuitively, it seems that any lightpath establishment heuristic which accounts for the forwarded traffic in a lightpath should provide better performance than a heuristic which only tries to maximize the single-hop traffic. This intuition led to the derivation of the current heuristic. The performance of this heuristic is found to be better than that of the previous heuristic (see Section VI).

Let $H_{s d}$ denote the minimum number of electronic hops needed to send a packet from source $s$ to destination $d$. Let 
$\Delta_{s d}$ denote the multihop traffic from source $s$ to destination $d$ under a fixed routing strategy. We start with a single optical channel on every fiber link (this is similar to the current Internet backbone which does not have any optical WDM switching). Initially, when no lightpaths have been established, $H_{s d}$ refers to the number of physical fiber hops that a packet would travel from source to destination. Let us assume that we get to add only one additional lightpath to this topology, such that in the new topology the average packet hop distance is minimum. The chosen lightpath needs to maximize the quantity $\Delta_{s d} \times\left(H_{s d}-1\right)$; provided sufficient network resources (transceivers and wavelengths) were available and this lightpath was established, this would correspond to the largest amount of multihop traffic that would be carried in one hop as opposed to using multiple hops, and this would result in the greatest minimization in the average packet hop distance.

We compute the quantity $\Delta_{s d} \times\left(H_{s d}-1\right)$ for all possible source-destination pairs; from all such values, choose the source-destination pair with the largest value, and establish a lightpath along the minimum-hop path between the two nodes. Recompute the $H_{s d}$ values; the $H_{s d}$ values get progressively smaller as new lightpaths are added. Repeat the entire process, progressively setting up new lightpaths, until resources (wavelengths or transceivers) run out. If a certain lightpath cannot be established, either due to transceiver constraints, or due to wavelength constraint, choose the next best alternative. This algorithm allows only a single lightpath to be established between any source-destination pair.

\section{RESOURCE BUDGETING IN OPTICAL NETWORK DESIGN}

This section discusses some of the network design principles that can be derived from the earlier ILP formulation. It is intuitive that, in a network with a very large number of transceivers per node, but with very few wavelengths per fiber and few fibers between node pairs, a large number of transceivers may be unused because some lightpaths may not be establishable due to fiber/wavelength constraints. Similarly, a network with few transceivers but a large number of available fibers and wavelengths may have a large number of wavelengths unutilized because the network is transceiver-constrained.

This mismatch in transceiver utilization versus wavelength utilization has a direct impact on the cost and the design of the network. The number of wavelengths supported in the network determines the cost of the switching equipment. ${ }^{5}$ Likewise, the number of transceivers per node determines the cost of the terminating equipment. We would like to balance these network resources, in order to maximize the utilizations of both the transceivers and the wavelengths in the network. Resource budgeting becomes an important issue when we attempt to optimize network design with constrained total cost.

\footnotetext{
${ }^{5} \mathrm{~A}$ WRS with $\delta$ input ports and $\delta$ output ports (including the ports used to connect to the local node used for electronic termination of lightpaths), supporting $W$ wavelengths, requires $2 W \delta \times \delta$ wavelength-insensitive optical switches for nonblocking operation. Increasing the number of fibers interconnecting two nodes increases $\delta$, and increasing $W$ increases the number of crosspoint switching elements required; thus, in both cases, the cost of the switching equipment would increase
}

A very simple and approximate calculation leads to some insights into the resource-budgeting problem. Given a physical topology, and a routing algorithm for lightpaths, we can determine the average length of a lightpath (in terms of the number of fiber links traversed by a lightpath, averaged over all source-destination pairs in the network); let the average length of a lightpath be denoted by $H_{P}$. If there are $M$ fiber links in the network, each supporting $W$ wavelengths, then the maximum number of lightpaths that can be supported is $M W / H_{P}$, assuming uniform utilization of wavelengths on all fiber links. ${ }^{6}$ Therefore, the number of transceivers per node should be approximately

$$
T_{i}=R_{i} \approx M W / N H_{P}
$$

in order to get a network with balanced loading across all wavelengths. Our optimization-based and heuristic-based network simulations on the NSFNET reinforce this conjecture (see Section VI).

\section{VIRTUAL TOPOLOGY RECONFIGURATION}

A major advantage of an optical network is that it may be able to reconfigure its virtual topology to adapt to changing traffic patterns. Some reconfiguration studies on optical networks have been reported before [3], [13], [19]; however, these studies assumed that the new virtual topology was known a priori, and were concerned with the cost and sequence of branch-exchange operations to transform from the original virtual topology to the new virtual topology. We propose a methodology to obtain the new virtual topology, based on optimizing a given objective function, as well as minimizing the changes required to obtain the new virtual topology from the current virtual topology. This approach would result in the minimum number of switch retunings, thus minimizing the number of disrupted lightpaths. Consequently, this approach also minimizes the time it takes to complete the reconfiguration process. Some discussions on the control mechanisms required to perform retuning of lightpaths can be found in [16].

The ILP formulation in Section II can help us derive new virtual topologies from existing virtual topologies. In the ideal situation, given a small change in the traffic matrix, we would prefer for the new virtual topology to be largely similar to the previous virtual topology, in terms of the constituent lightpaths and the routes for these lightpaths, i.e., we would prefer to minimize the changes in the number of WRS configurations needed to adapt from the existing virtual topology to the new virtual topology. More formally, it would be preferable if a large number of the $V_{i j}$ and the $p_{m n}^{i j}$ variables retain the same values in the two solutions, without compromising the quality of the solution (in terms of minimizing the average packet hop distance).

Let us consider the snapshot of two traffic matrices, $\Lambda_{s d}^{1}$ and $\Lambda_{s d}^{2}$, taken at two not-too-distant time instants. We assume that there is a certain amount of correlation between these two traffic

\footnotetext{
${ }^{6}$ Note, however, that in many cases, the routing on the network is dependent on the wavelength congestion in the network, and any static routing policy may not yield very accurate results.
} 
matrices. Given a certain traffic matrix, there may be many different virtual topologies, each of which has the same optimal value with regard to the objective function, i.e., (2). Usually, an optimizaton package will terminate after it has found the first such optimal solution. Our reconfiguration algorithm finds the virtual topology corresponding to $\Lambda_{s d}^{2}$ which matches "closest" with the virtual topology corresponding to $\Lambda_{s d}^{1}$ (based on our above definition of "closeness").

\section{A. Reconfiguration Algorithm}

We perform the following sequence of actions:

1) Generate linear formulations $\mathcal{F}(1)$ and $\mathcal{F}(2)$ corresponding to traffic matrices $\Lambda_{s d}^{1}$ and $\Lambda_{s d}^{2}$, respectively, based on the formulation in Section II.

2) Derive solutions $\mathcal{S}(1)$ and $\mathcal{S}(2)$, corresponding to $\mathcal{F}(1)$ and $\mathcal{F}(2)$, respectively. Denote the variables' values in $\mathcal{S}(1)$ as $V_{i j}(1), p_{m n}^{i j}(1), \lambda_{i j}^{s d}(1)$, and those in $\mathcal{S}(2)$ as $V_{i j}(2), p_{m n}^{i j}(2), \lambda_{i j}^{s d}(2)$, respectively. Let the value of the objective function for $\mathcal{S}(1)$ and $\mathcal{S}(2)$ be $O P T_{1}$ and $O P T_{2}$, respectively.

3) Modify $(\mathcal{F}(2))$ to $\left(\mathcal{F}^{\prime}(2)\right)$ by adding the new constraint

$$
\frac{1}{\sum_{s, d} \Lambda_{s d}^{2}} \sum_{i, j} \sum_{s, d} \lambda_{i j}^{s d}(2)=O P T_{2} .
$$

This ensures that all the virtual topologies generated by $\mathcal{F}^{\prime}(2)$ would be optimal with regard to the objective function.

The new objective function for $\mathcal{F}^{\prime}(2)$ is

$$
\text { Minimize : } \sum_{i j} \sum_{m n}\left|p_{m n}^{i j}(2)-p_{m n}^{i j}(1)\right| \text {. }
$$

We could also have used the following objective function:

$$
\text { Minimize : } \sum_{i j}\left|V_{i j}(2)-V_{i j}(1)\right| \text {. }
$$

Note that the mod operation, $|x|$, is a nonlinear function. If we assume that $p_{m n}^{i j}$ and $V_{i j}$ can only take on binary values, then (30) and (31) become linear, i.e., if $V_{i j}(1)=1$, then $\mid V_{i j}(2)-$ $V_{i j}(1) \mid \equiv\left(1-V_{i j}(2)\right)$; else if $V_{i j}(1)=0$, then $\mid V_{i j}(2)-$ $V_{i j}(1) \mid \equiv V_{i j}(2)$. Hence, $\mathcal{F}^{\prime}(2)$ may be solved directly.

Also, note that $\left|p_{m n}^{i j}(2)-p_{m n}^{i j}(1)\right| \Rightarrow\left|V_{i j}(2)-V_{i j}(1)\right|$. Therefore, (30) is a stronger condition than (31). Hence, we chose (30) for our simulation studies on reconfiguration at the end of Section VI.

\section{NUMERICAL EXAMPLES}

This section presents numerical examples of the network design problem, using the 14-node NSFNET T1 backbone (Fig. 1), the 15-node subset of the PACBELL network (Fig. 2), and a random biconnected topology with 20 nodes and 30 links, ${ }^{7}$ as our physical network topologies. Attempts to study larger network topologies had to be dropped because they overwhelmed our current computing resources. The links in

\footnotetext{
${ }^{7}$ Each node in the random topology had a physical nodal degree which was uniformly distributed between $2-6$.
}

the topologies are bidirectional, i.e., for each link, there is a pair of unidirectional fibers which carry transmissions in opposite directions and which join physically adjacent nodes, i.e., $P_{m n}=P_{n m}=1$. Each node consists of an optical WRS along with multiple transceivers for optical origination and termination of lightpaths. Each WRS is equipped with complete wavelength-conversion capabilities; in reality, it is possible that using sparse wavelength conversion [7], [8], [21] will be sufficient. The number of transmitters is assumed to be equal to the number of receivers, and is the same for all nodes.

The traffic matrix is randomly generated, such that a certain fraction $F$ of the traffic is uniformly distributed over the range $[0,(C / a)]$ and the remaining traffic is uniformly distributed over $[0,(C \times \Upsilon / a)]$, where $C$ is the lightpath channel capacity, $a$ is an arbitrary integer which may be one or greater, and $\Upsilon$ denotes the average ratio of traffic intensities between node-pairs with high traffic values and node-pairs with low traffic values. This model allows us to generate traffic patterns with varying characteristics.

In our first experiment, we used the CPLEX optimization package [1] to solve the complete virtual topology design formulation. Our results were averaged over 100 different traffic matrices for each of the three noted topologies. Initially, we solved the optimization formulation without any integer constraints [i.e., without (5) and (10)]; this is a linear program and therefore provides the lower bound for the result that can be achieved by any integer solution. Next, we solve the same optimization formulation including the integer constraints. We use the branch-and-bound algorithm in the CPLEX optimization package for solving the ILP. We found that, in spite of terminating the optimization after only two iterations of the branch-and-bound algorithm, the obtained solution was within $0.1 \%$ of the final value obtained by the fractional LP. This leads us to believe that a few iterations of the ILP is sufficient to generate high-quality solutions, and the number of iterations is a parameter that can be chosen by the network designer to strike a balance between the quality of the solution and the time taken to obtain the solution.

The number of variables and equations in the problem formulation increases very quickly as the number of nodes and links increases. On average, the time taken to solve the optimization was $5.72 \mathrm{~s}$ for the 14-node NSFNET topology, $26.114 \mathrm{~s}$ for the 15-node PACBELL topology, and $358.53 \mathrm{~s}$ for the random 20-node topology. All experiments were run on an otherwise unloaded HP-9000/778 running HPUX version 10 .

We studied resource utilization characteristics for the NSFNET topology. Figs. 3-5 plot system characteristics averaged over 25 different virtual topologies for the NSFNET, each corresponding to an independent traffic matrix, obtained with the parameters $C=1250, a=20, \Upsilon=10, F=0.7$, $\beta=0.8$, and $\alpha=2 . T_{i}$ and $R_{i}$ were assumed to be equal for all nodes, and were allowed values between 4 and 8 . $W$ was allowed to take values between 1 (no WDM) and 7 .

Fig. 3 plots the average packet hop distance for optimal virtual topologies for different number of transceivers per node, and different number of wavelengths in the system. The average hop distance in the network is a function of the number of lightpaths set up in the network, which directly depends on 


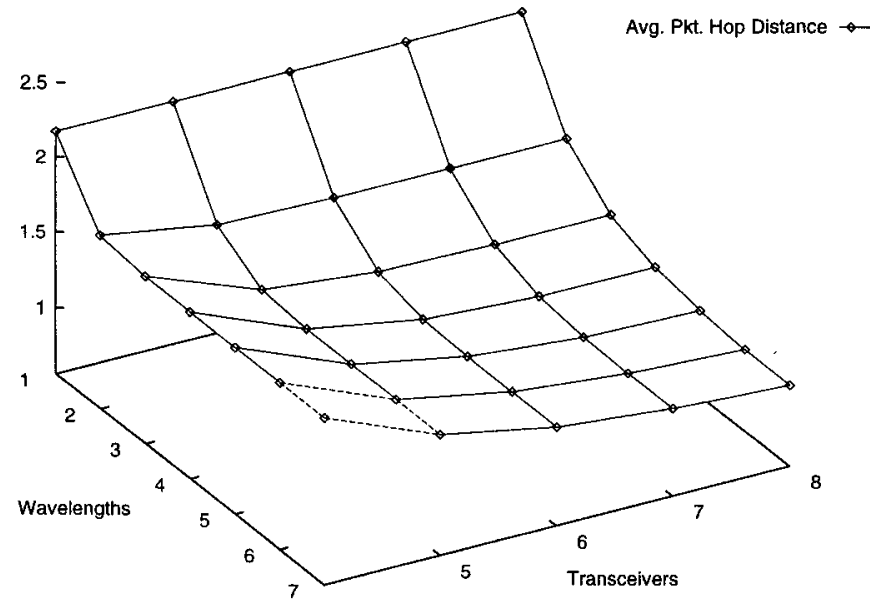

Fig. 3. Average packet hop distance for the optimal solution.

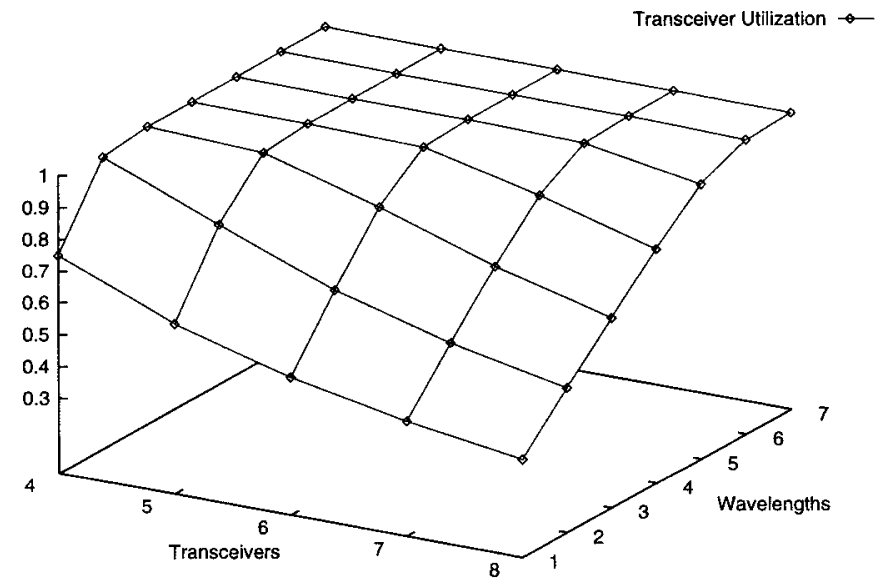

Fig. 4. Average transceiver utilization for the optimal solution.

the number of transceivers and wavelengths supported. The case corresponding to one wavelength in the system corresponds to a point-to-point network (no WDM). As expected, the average hop distance decreases with a balanced increase in the number of transceivers and wavelengths in the network. Increasing tranceivers without adding extra wavelengths marginally improves the quality of the solution. For more than six transceivers, and more than four wavelengths, the performance improvement in hop distance is marginal for the network in Fig. 1 for our current choice of network traffic. Given a different network topology, and a different traffic matrix, these results would be different.

Fig. 4 plots the transceiver utilization for different values of the number of wavelengths in the system, and number of transceivers at a node. Fig. 5 plots the wavelength utilization for the same set of experiments. As one would expect, Fig. 4 quantitatively demonstrates that the transceiver utilization decreases as the number of wavelengths is reduced and/or the number of transceivers is increased. Similarly, Fig. 5 demonstrates that the wavelength utilization decreases when the number of wavelengths is increased and/or the number of transceivers is reduced. These results confirm our original hypothesis that it is necessary to obtain the correct balance between transceivers and wavelengths in the system in order to have high utilizations of both these expensive resources. Given a cost constraint, re-

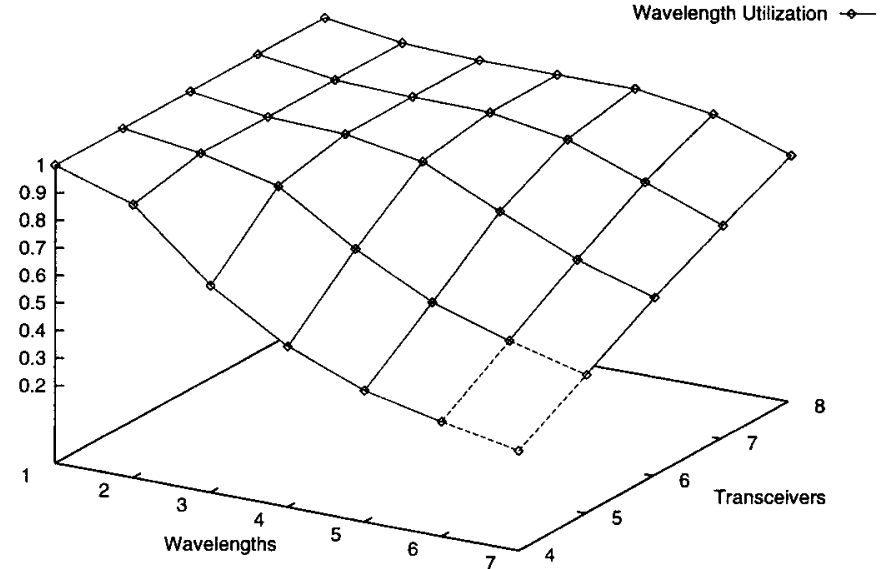

Fig. 5. Average wavelength utilization for the optimal solution.

TABLE I

Average Packet Hop Distance For Different Virtual Topology ESTABLISHMENT ALGORITHMS

\begin{tabular}{c|c|c|c|c|c|c|c}
\hline \multicolumn{7}{c}{ Integer Linear Problem Solution } \\
\hline Transc- & \multicolumn{7}{c}{ Wavelengths } \\
\cline { 2 - 8 } eivers & 1 & 2 & 3 & 4 & 5 & 6 & 7 \\
\hline 4 & 2.17 & 1.71 & 1.67 & 1.67 & 1.67 & 1.67 & 1.67 \\
5 & 2.17 & 1.58 & 1.39 & 1.36 & 1.36 & 1.36 & 1.36 \\
6 & 2.17 & 1.56 & 1.31 & 1.23 & 1.22 & 1.22 & 1.22 \\
7 & 2.17 & 1.56 & 1.29 & 1.19 & 1.15 & 1.14 & 1.14 \\
8 & 2.17 & 1.56 & 1.29 & 1.18 & 1.13 & 1.10 & 1.10 \\
\hline \multicolumn{7}{c}{ Maximizing Single-Hop Traffic Heuristic } \\
\hline Transc- & \multicolumn{7}{c}{ Wavelengths } \\
eivers & 1 & 2 & 3 & 4 & 5 & 6 & 7 \\
\hline 4 & 2.41 & 1.91 & 1.84 & 1.84 & 1.84 & 1.84 & 1.84 \\
5 & 2.41 & 1.77 & 1.52 & 1.47 & 1.46 & 1.46 & 1.46 \\
6 & 2.41 & 1.75 & 1.41 & 1.31 & 1.37 & 1.27 & 1.27 \\
7 & 2.41 & 1.74 & 1.39 & 1.26 & 1.20 & 1.20 & 1.17 \\
8 & 2.41 & 1.74 & 1.39 & 1.24 & 1.17 & 1.14 & 1.12 \\
\hline \hline \multicolumn{7}{|c|}{ Maximizing Multihop Traffic Heuristic } \\
\hline Transc- & 1 & 2 & 3 & 4 & 5 & 6 & 7 \\
\cline { 2 - 7 } eivers & 1 & 1.87 & 1.80 & 1.79 & 1.79 & 1.79 & 1.79 \\
\hline 4 & 2.41 & 1.87 & 1.49 & 1.44 & 1.44 \\
5 & 2.41 & 1.72 & 1.50 & 1.45 & 1.44 & 1.27 \\
6 & 2.41 & 1.70 & 1.41 & 1.30 & 1.27 & 1.26 & 1.27 \\
7 & 2.41 & 1.69 & 1.39 & 1.26 & 1.20 & 1.18 & 1.17 \\
8 & 2.41 & 1.69 & 1.38 & 1.24 & 1.16 & 1.13 & 1.12 \\
\hline \hline
\end{tabular}

source-budgeting tradeoff becomes an important issue, in order to not underutilize transceivers and wavelengths in the system.

Comparing Figs. 3-5 together, it is clear that, for the current network setting, the choice of six transceivers and four wavelengths is a very good one since it yields an average hop distance close to unity while achieving nearly $100 \%$ utilization of transceivers and wavelengths. A network designer may use our approach to similarly determine the best configuration in which to operate his/her network.

Table I tabulates the average hop distance for the two heuristic approaches as compared to the optimal solution obtained in Fig. 3. The same sample of 25 traffic matrices were used to evaluate the performance of the heuristics. Fig. 6 also plots these performance results for a four-wavelength system. As expected, the average hop distance decreases with an increase in 


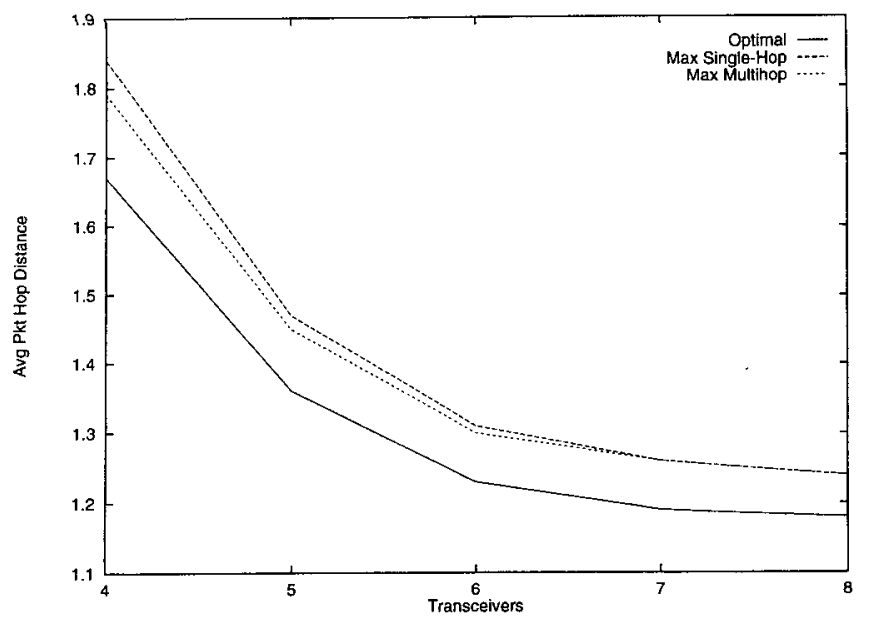

Fig. 6. Comparison of heuristic algorithms for a four-wavelength network.

the number of transceivers in the system, with the heuristics performing a little poorly relative to the ILP's optimal solution which can be treated as a lower bound. Also, the heuristic which maximizes the multihop traffic is found to perform a little better than the heuristic which maximizes single-hop traffic for smaller number of transceivers.

Although we assumed complete wavelength conversion in all nodes of the network, using a simple first-fit wavelength assignment strategy, we found that we needed only two wavelength convertors, for a given traffic matrix on the NSFNET topology. This supports the theory that sparse wavelength conversion should be sufficient in most networks of interest. A complete and detailed description of an example embedding can be found in [2].

We study the performance of the reconfiguration algorithm proposed in Section V-A. We generate two sequences of 25 traffic matrices, with the same set of statistical parameters as used before. In the first sequence, exactly $20 \%$ of the entries in successive traffic matrices in the sequence are forced to differ. In the second sequence, $80 \%$ of the entries differ. The traffic sequence is created by generating an initial traffic matrix, and then swapping a fraction (either $20 \%$ or $80 \%$ depending on the chosen sequence) of nondiagonal entries in the traffic matrix. The algorithm in Section V-A was applied to this traffic sequence, in order to generate virtual topologies in a network with eight transceivers per node and eight wavelengths per fiber. Fig. 7 plots the fraction of lightpath additions and deletions as observed over the sequence of 25 traffic matrices, for $20 \%$ and $80 \%$ changes in the traffic matrix. The fraction of common lightpaths between two successive traffic matrices remains fairly uniform throughout the entire sequence. As expected, the number of deleted lightpaths and added lightpaths increases when the difference between consecutive traffic matrices gets larger. The proposed algorithm, however, minimizes the number of disrupted lightpaths due to the reconfiguration.

\section{CONCLUSION}

This paper presented an integer linear programming (ILP) formulation to derive a minimal-hop-distance solution to the virtual topology design problem in a wavelength-routed optical

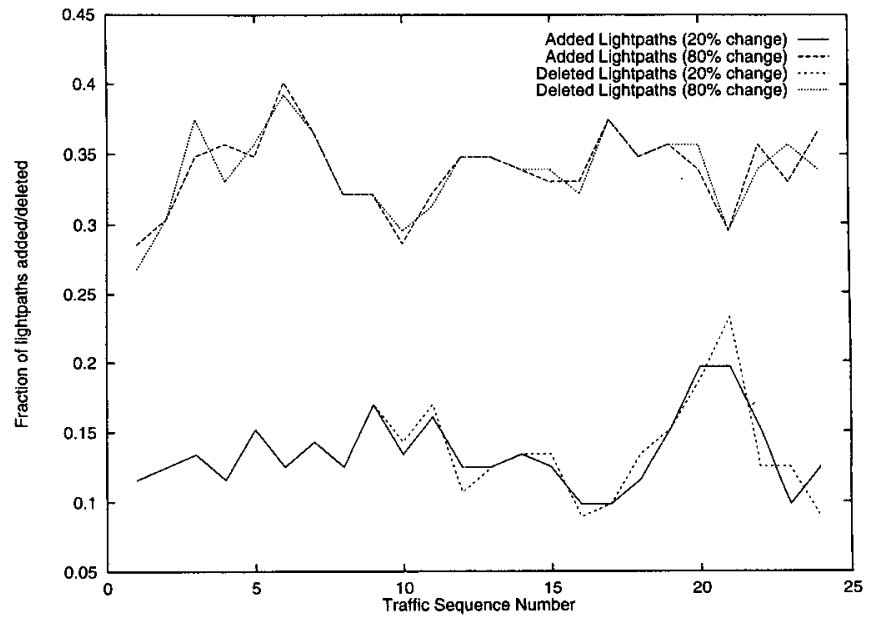

Fig. 7. Reconfiguration statistics.

network, in the absence of wavelength-continuity constraints. The problem formulation is general, and can be used to derive a complete virtual topology solution, including choice of the constituent lightpaths, routes for these lightpaths, and intensity of packet flows through these lightpaths. We observed that adding the wavelength-continuity constraints and queueing delays makes the problem formulation more complex. We used many simplifying assumptions in order to engineer the problem to a tractable form. We also proposed two simple heuristics and demonstrated that these heuristics perform well with respect to the optimal solution.

We studied resource-budgeting tradeoffs in the allocation of transceivers per node, and wavelengths per fiber. A simple computation in Section IV (28) provided an approximate bound regarding the number of transceivers that can be supported in a network with $W$ wavelengths. We demonstrated how we can equip the network with an optimal balance of transceivers and wavelengths, in order to derive minimal-hop-distance solutions, along with high utilization of both transceivers and wavelengths.

We proposed an exact reconfiguration procedure which, for a changed traffic matrix, searches through all possible optimal virtual topologies, in order to obtain a solution which shares the maximum number of lightpaths with the previous virtual topology. The solution to the reconfiguration algorithm generates a virtual topology which minimizes the amount of switch retunings that needs to be performed, in order to adapt the virtual topology to the new traffic matrix. We believe that reconfiguration in wide-area optical networks is an important issue that needs to be studied further. We would prefer to have algorithms, which can perform network reconfiguration locally, without a global knowledge of the network state. The sequence of actions that need to be performed to effect a lightpath reconfiguration is an important problem in network management, and is an open problem for further research.

\section{ACKNOWLEDGMENT}

The authors would like to thank the reviewers and the Editor for their constructive criticism which greatly helped to improve the paper. 


\section{REFERENCES}

[1] ILOG CPLEX Division. (1998) CPLEX optimization package. [Online]. Available: www.cplex.com

[2] D. Banerjee, "Design and analysis of wavelength-routed optical networks," Ph.D. dissertation, Univ. Calif., Davis, CA, 1996.

[3] D. Bienstock and O. Gunluk, "A degree sequence problem related to network design," Networks, vol. 24, no. 4, pp. 195-205, July 1994.

[4] B. Mukherjee, Optical Communication Networks. New York: McGraw Hill, 1997.

[5] C. Chen and S. Banerjee, "Optical switch configuration and lightpath assignment in wavelength routing multihop lightwave networks," in Proc. IEEE INFOCOM, Boston, MA, June 1995, pp. 1300-1307.

[6] I. Chlamtac, A. Ganz, and G. Karmi, "Lightnets: Topologies for high speed optical networks," J. Lightwave Technol., vol. 11, pp. 951-961, May/June 1993.

[7] J. Iness, "Efficient use of optical components in WDM-based optical networks," Ph.D. dissertation, Univ. Calif., Davis, CA, 1997.

[8] J. Iness and B. Mukherjee, "Sparse wavelength conversion in wavelength-routed WDM networks," Photon. Network Commun., vol. 1, no. 3, pp. 183-502, Nov. 1999.

[9] "Special Issue on Multiwavelength Optical Technology and Networks," J. Lightwave Technology, vol. 14, June 1996.

[10] "Special Issue on Optical Networks," IEEE J. Select. Areas Commun. vol. 14, June 1996.

[11] "Special Issue on High Capacity Optical Transport Networks," IEEE J. Select. Areas Commun., vol. 16, no. 7, Sept. 1998.

[12] J. F. P. Labourdette, "Rearrangeability techniques for multihop lighwave networks and application to distributed ATM switching systems," Ph.D. dissertation, Columbia Univ., New York, 1991.

[13] J. F. P. Labourdette, G. W. Hart, and A. S. Acampora, "Branch-exchange sequences for reconfiguration of lightwave networks," IEEE Trans. Commun., vol. 42, pp. 2822-2832, Oct. 1994.

[14] K. C. Lee and V. O. K. Li, "A wavelength convertible optical network," J. Lightwave Technol., vol. 11, pp. 962-970, May/June 1993.

[15] B. Mukherjee, D. Banerjee, S. Ramamurthy, and A. Mukherjee, "Some principles for designing a wide-area optical network," IEEE/ACM Trans. Networking, vol. 4, pp. 684-696, Oct. 1996.

[16] R. Ramaswami and A. Segall, "Distributed network control for wavelength routed optical networks," IEEE/ACM Trans. Networking, vol. 5, pp. 936-943, Dec. 1997.

[17] R. Ramaswami and K. Sivarajan, Optical Networks: A Practical Perspective. San Mateo, CA: Morgan Kaufmann, 1998.

[18] - "Design of logical topologies for wavelength-routed optical networks," IEEE J. Select. Areas Commun., vol. 14, pp. 840-851, June 1996.

[19] G. N. Rouskas and M. H. Ammar, "Dynamic reconfiguration in multihop WDM networks," J. High Speed Networks, vol. 4, no. 3, pp. 221-238, 1995.

[20] T. E. Stern and K. Bala, Multiwavelength Optical Networks: A Layered Approach. Reading, MA: Addison Wesley, 1999.
[21] S. Subramaniam, M. Azizoglu, and A. K. Somani, "All-optical networks with sparse wavelength conversion," IEEE/ACM Trans. Networking, vol. 4, pp. 544-557, Aug. 1996.

[22] Z. Zhang and A. Acampora, "A heuristic wavelength assignment algorithm for multihop WDM networks with wavelength routing and wavelength reuse," IEEE/ACM Trans. Networking, vol. 3, pp. 281-288, June 1995.

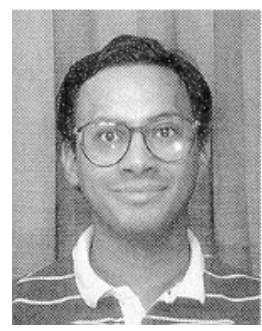

Dhritiman Banerjee received the B. Tech. (Hons) degree from the Indian Institute of Technology, Madras, India, in 1992, and the M.S. and Ph.D. degrees in computer science from the University of California, Davis, in 1994 and 1996, respectively.

He has published extensively in optical networking, with over a dozen publications and multiple patents. He is on the review committee for many networking journals and currently serves as the Guest Editor for Optical Networks Magazine. He has worked at Telesis Technology Labs, Tandem Computers, and Hewlett Packard in the past, and is currently associated with the Optical Networking Research Division of Bell Labs, Lucent Technologies, Holmdel, NJ.

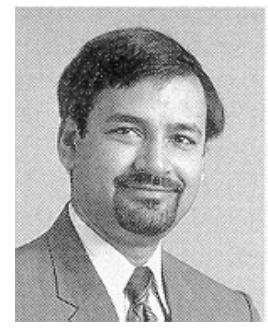

Biswanath Mukherjee ('' $82-M^{\prime} 87$ ) received the B.Tech. (Hons) degree from the Indian Institute of Technology, Kharagpur, India, in 1980 and the Ph.D. degree from the University of Washington, Seattle, in June 1987.

At the University of Washington, he held a GTE Teaching Fellowship and a General Electric Foundation Fellowship. In July 1987, he joined the University of California, Davis, where he has been Professor of Computer Science in July, 1995, and Chairman of Computer Science in September, 1997. He serves on the editorial boards of the IEEE/ACM TRANSACTIONS ON NETWORKING, IEEE Network, ACM/Baltzer Wireless Information Networks (WINET), Journal of High-Speed Networks, Photonic Network Communications, and Optical Network Magazine. He also served as Editor-at-Large for optical networking and communications for the IEEE Communications Society. He is author of the textbook Optical Communication Networks (New York: McGraw-Hill,1997), a book which received the Association of American Publishers, Inc.'s 1997 Honorable Mention in Computer Science. His research interests include lightwave networks, network security, and wireless networks.

Dr. Mukherjee served as the Technical Program Chair of the IEEE INFOCOM'96 conference. He is co-recipient of paper awards presented at the 1991 and 1994 National Computer Security Conferences. 